\title{
Androgen deprivation therapy for prostate cancer and the risk of hospitalisation for community-acquired pneumonia
}

\author{
Blánaid M Hicks, ${ }^{1,2}$ Hui Yin, ${ }^{1}$ Franck Bladou, ${ }^{3,4}$ Pierre Ernst, ${ }^{1,5}$ Laurent Azoulay ${ }^{1,2,6}$
}

\begin{abstract}
- Additional material is
published online only. To view please visit the journal online (http://dx.doi.org/10.1136/ thoraxjn-2016-209512).
\end{abstract}

${ }^{1}$ Centre for Clinical Epidemiology, Lady Davis Institute, Jewish General Hospital, Montréal, Quebec, Canada

${ }^{2}$ Department of Epidemiology, Biostatistics and Occupational Health, McGill University,

Montréal, Quebec, Canada ${ }^{3}$ Department of Urology, Jewish General Hospital, Montréal, Quebec, Canada

${ }^{4}$ Division of Urology, McGill University, Montréal, Quebec, Canada

${ }^{5}$ Department of Medicine, McGill University, Montréal, Quebec, Canada

${ }^{6}$ Gerald Bronfman Department of Oncology, McGill University, Montréal, Quebec, Canada

\section{Correspondence to}

Dr Laurent Azoulay, Center for Clinical Epidemiology, Lady Davis Institute, Jewish General Hospital, 3755 Côte SainteCatherine, H-425.1, Montreal, Quebec, Canada H3T 1E2; laurent.azoulay@mcgill.ca

Received 29 September 2016 Revised 8 November 2016 Accepted 14 November 2016 Published Online First 16 December 2016

\section{SLinked}

- http://dx.doi.org/10.1136/ thoraxjnl-2016-209773

CrossMark

To cite: Hicks BM, Yin $\mathrm{H}$ Bladou F, et al. Thorax 2017:72:628-634.

\section{ABSTRACT}

Background Androgens have been shown to influence both the immune system and lung tissue, raising the hypothesis that androgen deprivation therapy (ADT) for prostate cancer may increase the risk of pneumonia. Thus, the aim of this study was to determine whether ADT is associated with an increased risk of hospitalisation for community-acquired pneumonia in patients with prostate cancer.

Methods This was a population-based cohort study using the United Kingdom Clinical Practice Research Datalink linked to the Hospital Episode Statistics repository. The cohort consisted of 20310 men newly diagnosed with non-metastatic prostate cancer between 1 April 1998 and 31 March 2015. Time-dependent Cox proportional hazards models were used to estimate adjusted HRs and $95 \%$ Cls for hospitalisation for community-acquired pneumonia associated with current and past use of ADT compared with non-use.

Results During a mean follow-up of 4.3 years, there were 621 incident hospitalisations for communityacquired pneumonia (incidence rate: 7.2/1000 personyears). Current ADT use was associated with an $81 \%$ increased risk of hospitalisation for community-acquired pneumonia (12.1 vs 3.8 per 1000 person-years, respectively; HR $1.81,95 \% \mathrm{Cl} 1.47$ to 2.23 ). The association was observed within the first six months of use (HR $1.73,95 \% \mathrm{Cl} 1.23$ to 2.42 ) and remained elevated with increasing durations of use ( $\geq 25$ months; HR $1.79,95 \% \mathrm{Cl} 1.39$ to 2.30 ). In contrast, past ADT use was not associated with an increased risk (HR 1.23, $95 \% \mathrm{Cl} 0.95$ to 1.60$)$.

Conclusions The use of ADT is associated with an increased risk of hospitalisation for community-acquired pneumonia in men with prostate cancer.

\section{INTRODUCTION}

Androgen deprivation therapy (ADT) is the mainstay treatment for patients with prostate cancer, of which the benefits on disease progression and survival for patients with high risk and metastatic disease have been known for many years. ${ }^{1}$ However, recent years have seen an increase in the use of ADT across all stages of disease, including low-risk patients for whom the benefits are unclear. ${ }^{1}$ In the UK, ADT may be used in different settings; as a neoadjuvant or adjuvant treatment (for at least 6 months up to 3 years) with radiation therapy or radical prostatectomy, in patients with intermediate and advanced disease, as well as in patients with high-risk localised disease. ${ }^{2}$ In patients with

\section{Key messages}

What is the key question?

- Does androgen deprivation therapy for prostate cancer increase the risk of hospitalisation for community-acquired pneumonia?

What is the bottom line?

- The use of androgen deprivation therapy is associated with an increased risk of hospitalisation for community-acquired pneumonia; however, past use of androgen deprivation therapy is not associated with an increased risk.

\section{Why read on?}

- This study represents the most detailed assessment to date of this important adverse outcome, which is of particular concern among those with cancer for whom severe pneumonia can result in high mortality rates.

advanced and metastatic disease, ADT is typically continued indefinitely until evidence of treatment resistance. ${ }^{3}$ While the use of ADT has increased in recent decades, there is mounting evidence that this therapy may be associated with a number of adverse effects, such as loss of bone mineral density, metabolic syndrome and cardiovascular outcomes. ${ }^{4}$ There is also some evidence that ADT may have effects both on immune function and lung tissue, thus raising the possibility that its use may increase the risk of infections, such as pneumonia. ${ }^{5-7}$

Androgens have been shown to play an important role on the immune system. Indeed, androgen receptors are expressed on a number of immunological cells, ${ }^{8}$ and androgens have been reported to increase neutrophil production. ${ }^{9-11}$ Furthermore, in mice, androgen depletion impaired the adaptive immune response, rendering mice more susceptible to microbial infection. ${ }^{12}{ }^{13}$ It was also shown that androgen deprivation may influence the risk of lower respiratory tract infections via morphological and biochemical changes to cell membranes within the lung. ${ }^{67}$

To date, only two observational studies have investigated the association between ADT and pneumonia, but reported conflicting findings. ${ }^{14} 15$ These studies had a number of methodological limitations, including potential misclassification of 
hospital-acquired versus community-acquired pneumonia, ${ }^{14} 15$ limiting the analysis to one type of $\mathrm{ADT}^{14}$ and immortal time bias leading to an underestimation of the association. ${ }^{14} 15$

Community-acquired pneumonia is of particular concern in older adults, ${ }^{16}$ particularly among those with cancer for whom severe pneumonia can result in high mortality rates. ${ }^{17}$ Thus, the objective of this study was to determine whether the use of ADT is associated with an increased risk of hospitalisation for community-acquired pneumonia in patients with prostate cancer.

\section{METHODS}

\section{Data sources}

This study was conducted using the UK Clinical Practice Research Datalink (CPRD) linked to the Hospital Episode Statistics (HES) repository. The CPRD is a large computerised database of longitudinal patient records, comprising primary care records for $>14$ million patients from $>700$ general practices. The CPRD includes information on demographic characteristics, lifestyle data (such as smoking status and alcohol use), medical diagnoses and procedures (coded using the Read code classification ${ }^{18}$ ) and prescriptions written by general practitioners (coded according to the UK Prescription Pricing Authority Dictionary ${ }^{19}$ ). The data recorded in CPRD have been shown to be of high quality. ${ }^{20} 21$

The HES repository contains all inpatient and day case admission information, including primary and secondary diagnoses (based on the International Classification of Disease codes (ICD-10 codes)) and related hospital-based procedures. The linkage of CPRD to HES is possible from April 11997 onwards and is limited to English general practices that have consented to the linkage scheme (currently representing $75 \%$ of all English practices). ${ }^{22}$

\section{Study cohort}

We assembled a cohort of men, at least 40 years of age, who were newly diagnosed with prostate cancer between 1 April 1998 and 31 August 2015. Cohort entry was defined by the date of the first prostate cancer diagnosis. All patients were required to have at least one year of medical history in the CPRD. We excluded patients diagnosed with metastatic disease (defined as metastases to the bones, lymph nodes, viscerally and other sites), as well as those with a history of ADT use (as this indicates inaccuracy in the recording of the prostate cancer diagnosis and removes prevalent ADT users). Additionally, all patients who received chemotherapy or radiation therapy in the year prior to cohort entry were excluded, as were those with prescriptions for anti-TB drugs (in the year before cohort entry) and those with a previous diagnosis of HIV. Finally, all patients hospitalised for any cause (with a length of stay $\geq 2$ days) in the 30 days before cohort entry were excluded to ensure that early pneumonia events occurring after cohort entry were likely to be community-acquired pneumonia and not the result of a previous hospitalisation.

All patients were followed from cohort entry until a first hospitalisation for community-acquired pneumonia (defined below) or were censored upon a diagnosis for hospital-acquired pneumonia (defined below), receipt of chemotherapy, death from any cause, end of registration with the general practice or end of the study period (31 August 2015), whichever occurred first.

\section{Exposure definition}

Exposure to ADT included gonadotropin-releasing hormone (GnRH) agonists (leuprolide, buserelin, goserelin, triptorelin), oral antiandrogens (cyproterone acetate, flutamide, bicalutamide, nilutamide), oestrogens (diethylstilbestrol, estramustine) and bilateral orchiectomy. A time-dependent exposure definition was used to classify each person-day of follow-up as exposed or unexposed to ADT. Based on this definition, ADT use was classified as either current use, past use or non-use. Current use was defined as use at the time of the event; past use was defined as use of ADT prior to but not at the time of the event; and non-use was defined as no use up until the time of the event. The latter category served as the reference category in all analyses. Due to the persistence of hypogonadism after treatment discontinuation, ${ }^{23} 24$ we considered a 180-day residual effect period for GnRH agonists and a 30-day period for oral antiandrogens and oestrogens. Therefore, patients were considered continuously exposed if the duration of one prescription plus the residual effect period overlapped with the date of the next prescription. Patients who underwent bilateral orchiectomy were considered continuously exposed from the date of surgery until the end of follow-up.

\section{Outcome definition}

A hospitalisation for community-acquired pneumonia was defined as a hospital admission preceded by an emergency department visit, with a primary (most responsible) diagnosis of pneumonia within the first two days of hospital admission (to minimise the inclusion of hospital-acquired pneumonia). The date of the hospital admission was considered the event date. Hospital-acquired pneumonia was considered as a pneumonia diagnosis $>2$ days after admission, as well as pneumonia diagnosed up to 7 days after a hospital discharge.

\section{Potential confounders}

All models were adjusted for the following potential confounders measured at cohort entry: age, year of cohort entry, body mass index (BMI), smoking status, alcohol-related disorders, pre-diagnostic prostate-specific antigen (PSA) levels (closest measurement before cohort entry) and seasonality (with highrisk season defined as 1 October to $31 \mathrm{March}$ ). ${ }^{25} 26$ The models also included previous cancer diagnoses (excluding nonmelanoma skin cancers), COPD, asthma, bronchitis and bronchiectasis, in the year before cohort entry. Finally, the models were adjusted for pharmacological exposures measured in the year prior to cohort entry including immunosuppressive agents, inhaled bronchodilators, oral and inhaled corticosteroids, oral antibiotics, as well receipt of influenza and pneumococcal vaccinations.

\section{Statistical analyses}

Descriptive statistics were used to summarise the characteristics of the cohort. Crude incidence rates of hospitalisation for community-acquired pneumonia, with 95\% CIs based on the Poisson distribution, were calculated for each exposure group. Time-dependent Cox proportional hazards models were used to estimate crude and adjusted HRs and 95\% CIs of hospitalisation for community-acquired pneumonia associated with the current and past use of ADT compared with non-use. All models were adjusted for the potential confounders listed above.

\section{Secondary analyses}

We conducted two secondary analyses. First, we assessed whether there was a duration-response relationship between current use of ADT and the risk community-acquired pneumonia according to five predefined duration categories: $<6$ months, 6-12 months, 13-18 months, 19-24 months and $\geq 25$ months. 
In addition, duration of use was modelled as a continuous variable using a restricted cubic spline model with five knots to produce a smooth curve of the HR as a function of duration of use. $^{27} 28$ Second, we investigated whether the risk of hospitalisation for community-acquired pneumonia varied according to current use of specific types of ADT. This was assessed by classifying current use of ADT into one of six mutually exclusive categories: GnRH agonists alone, GnRH agonists and oral antiandrogens, oral antiandrogens alone, bilateral orchiectomy, oestrogens alone and other treatment combinations.

\section{Sensitivity analyses}

We conducted six sensitivity analyses to assess the robustness of our findings. First, the length of the residual effect period for GnRH agonists has been shown to be highly variable and dependent on factors such as age and treatment duration. ${ }^{23} 24$ Therefore, to account for this variability, we considered residual effect periods of 90 and 365 days for GnRH agonists. Second, we used a less restrictive outcome definition for a hospitalisation for community-acquired pneumonia to include those with an ICD-10 diagnosis code recorded in the primary as well as in the secondary position within the first two days of hospital admission. Third, we repeated the primary analysis by using a stricter definition for hospital-acquired pneumonia by censoring patients hospitalised for pneumonia in the 14 days (instead of 7 days) after a hospital discharge. Fourth, we repeated the primary analysis using multiple imputation for variables with missing values (including smoking, BMI and PSA levels). ${ }^{29} 30$ Fifth, to further minimise residual confounding, we conducted an analysis stratified on high-dimensional propensity score deciles (described in online supplementary method 1). Finally, to address potential residual time-dependent confounding over the 18-year follow-up period, the primary analysis was repeated using a marginal structural Cox proportional hazards model, a method designed to adjust for time-dependent confounding associated with time-varying exposures. ${ }^{31} 32$ All covariates mentioned previously were measured during follow-up, along with the addition of prostate cancer disease markers (ie, radical prostatectomy, incident metastasis (lymph node, bone, visceral and other), and the use of oral bisphosphonates, chemotherapy and radiation therapy). This is described in detail in online supplementary method 2. All statistical analyses were conducted using SAS V.9.4 (SAS institute, Cary, North Carolina, USA).

\section{RESULTS}

The cohort included 20310 patients (figure 1), which were followed for a mean (SD) of 4.3 (3.4) years. Overall, there were 621 incident community-acquired pneumonia events during 86301 person-years of follow-up, generating a crude incidence rate of 7.2 (95\% CI 6.6 to 7.8 ) per 1000 person-years. A total of $11912(58.7 \%)$ patients used ADT during the follow-up period, for a median (IQR) duration of $1.6(0.8-3.3)$ years.

Table 1 presents baseline characteristics for the cohort and by ADT use in the first six months of follow-up. ADT users tended to be older, had higher BMI and were less likely to be smokers. Furthermore, ADT users tended to have higher PSA levels at baseline and were more likely to have certain comorbidities including chronic obstructive pulmonary disorder and bronchitis. Finally, ADT users were more likely to have used certain medications including inhaled bronchodilators, corticosteroids (inhaled and oral), oral antibiotics and to have been vaccinated for influenza in the year before cohort entry.

The results of primary and secondary analyses are presented in table 2. Compared with no ADT use, current use of ADT was associated with an $81 \%$ increased risk of hospitalisation for community-acquired pneumonia (12.1 vs 3.8 per 1000 personyears; HR 1.81, 95\% CI 1.47 to 2.23 ). ADT was associated with an increased risk of hospitalisation for community-acquired pneumonia regardless of treatment duration, although the highest HR was observed between 13 and 18 months (HR 2.45, $95 \%$ CI 1.41 to 3.67$)$. In the restricted cubic spline analysis, the risk of pneumonia was increased with $<6$ months of ADT use and continued to increase with increasing durations of use (figure 2). Similarly, all types of ADT were associated with an increased risk of hospitalisation for community-acquired pneumonia, but with the highest HRs observed for current use of oestrogens alone (HR 4.50, 95\% CI 1.82 to 11.17), followed by bilateral orchiectomy (HR 2.32, 95\% CI 1.02 to 5.20) and then GnRH agonists and oral antiandrogens used in combination therapy (HR 2.18, 95\% CI 1.56 to 3.03). In contrast, past
Figure 1 Study flow diagram describing the construction of the study cohort.

\begin{tabular}{|c|c|c|c|}
\hline \multirow[t]{2}{*}{30,229} & \multicolumn{2}{|c|}{$\begin{array}{l}\text { Patients diagnosed with } \\
\text { prostate cancer between } \\
\text { April 1, } 1998 \text { and August 31, } \\
2015\end{array}$} & \\
\hline & 6966 & $\begin{array}{l}\text { Excluded } \\
3681 \\
236 \\
8 \\
2108 \\
933\end{array}$ & $\begin{array}{l}<1 \text { year of medical history before cohort entry } \\
\text { Date inconsistencies } \\
<40 \text { years of age } \\
\text { Previous use of androgen deprivation therapy } \\
\text { Metastatic disease at diagnosis }\end{array}$ \\
\hline \multirow[t]{2}{*}{23,263} & \multicolumn{2}{|c|}{ Patients with prostate cancer } & \\
\hline & 2953 & $\begin{array}{l}\text { Excluded } \\
209 \\
85 \\
3 \\
14 \\
2617 \\
25\end{array}$ & $\begin{array}{l}\text { Previous chemotherapy } \\
\text { Previous radiation therapy } \\
\text { Prescriptions of anti-tuberculosis drugs in the year prior } \\
\text { Previous diagnosis of HIV } \\
\text { Hospitalization within } 30 \text { days before cohort entry } \\
\text { No follow-up }\end{array}$ \\
\hline
\end{tabular}

20,310 Study cohort 
Table 1 Baseline demographic and clinical characteristics of the cohort and stratified by use of androgen deprivation therapy (ADT)

\begin{tabular}{|c|c|c|c|}
\hline \multirow[b]{2}{*}{ Characteristic } & \multirow{2}{*}{$\begin{array}{l}\text { Entire cohort } \\
\mathrm{N}, \%\end{array}$} & \multicolumn{2}{|c|}{ ADT use during follow-up* } \\
\hline & & Any ADT, \% & No ADT, \% \\
\hline Total & 20310 & $10250(50.5)$ & $10060(49.5)$ \\
\hline Age, years (mean, SD) & $71.2(9.1)$ & $72.8(8.2)$ & $68.6(9.1)$ \\
\hline \multicolumn{4}{|l|}{ Body mass index, $\mathrm{n}(\%)$} \\
\hline$<25 \mathrm{~kg} / \mathrm{m}^{2}$ & $6386(31.4)$ & $3257(31.8)$ & $3129(31.1)$ \\
\hline $25-30 \mathrm{~kg} / \mathrm{m}^{2}$ & $9069(44.7)$ & $4397(42.9)$ & $4672(46.4)$ \\
\hline$\geq 30.0$ & 3785 (18.6) & 2001 (19.5) & $1784(17.7)$ \\
\hline Unknown & $1070(5.3)$ & $595(5.8)$ & $475(4.7)$ \\
\hline \multicolumn{4}{|l|}{ Smoking status, $\mathrm{n}(\%)$} \\
\hline Current & $2249(11.1)$ & $1215(11.9)$ & $1034(10.3)$ \\
\hline Past & $7880(38.8)$ & $4147(40.6)$ & $3733(37.1)$ \\
\hline Never & $9639(47.5)$ & $4596(44.8)$ & $5043(50.1)$ \\
\hline Unknown & $542(2.7)$ & $292(2.9)$ & $250(2.5)$ \\
\hline Alcohol-related disorders, n (\%) & $1899(9.4)$ & $937(9.1)$ & $962(9.6)$ \\
\hline \multicolumn{4}{|l|}{ Prostate-specific antigen, $\mathrm{n}(\%)$} \\
\hline$<4 \mathrm{ng} / \mathrm{mL}$ & $1192(5.9)$ & $250(2.4)$ & $942(9.4)$ \\
\hline $4-10 \mathrm{ng} / \mathrm{mL}$ & $6459(31.8)$ & $1814(17.7)$ & $4645(46.2)$ \\
\hline$\geq 10 \mathrm{ng} / \mathrm{mL}$ & $9704(47.8)$ & $6673(65.1)$ & $3031(30.1)$ \\
\hline Unknown & $2955(14.6)$ & $1513(14.8)$ & $1442(14.3)$ \\
\hline High-risk season, n (\%) & $10203(50.2)$ & $5079(49.6)$ & $5124(50.9)$ \\
\hline Previous cancer, n (\%) & 2348 (11.6) & $1223(11.9)$ & $1125(11.2)$ \\
\hline COPD, n (\%) & $2617(12.9)$ & $1447(14.1)$ & $1170(11.6)$ \\
\hline Asthma, n (\%) & $2366(11.7)$ & 1160 (11.3) & $1206(12.0)$ \\
\hline Bronchitis, n (\%) & $1540(7.6)$ & $854(8.3)$ & $686(6.8)$ \\
\hline Bronchiectasis, n (\%) & $41(0.2)$ & $18(0.2)$ & $23(0.2)$ \\
\hline Immunosuppressive agents, n (\%) & $167(0.8)$ & $81(0.8)$ & $86(0.9)$ \\
\hline Inhaled bronchodilators, n (\%) & $2519(12.4)$ & $1341(13.1)$ & $1178(11.7)$ \\
\hline Inhaled corticosteroids, n (\%) & $2003(9.9)$ & $1067(10.4)$ & $936(9.3)$ \\
\hline Oral corticosteroids, n (\%) & $1182(5.8)$ & $625(6.1)$ & $557(5.5)$ \\
\hline Non-topical antibiotics, n (\%) & $8719(42.9)$ & $4479(43.7)$ & $4240(42.2)$ \\
\hline Influenza vaccines, n (\%) & 10444 (51.4) & $6040(58.9)$ & $4404(43.8)$ \\
\hline Pneumococcal vaccines, $\mathrm{n}(\%)$ & $1258(6.2)$ & $691(6.7)$ & $567(5.6)$ \\
\hline
\end{tabular}

${ }^{*}$ ADT use in the first six months during follow-up.

use of ADT was not associated with an increased risk of hospitalisation for community-acquired pneumonia compared with non-use (HR 1.23, 95\% CI 0.95 to 1.60 ).

\section{Sensitivity analyses}

The sensitivity analyses are presented in figure 3 and online supplementary tables S1-S7. Overall, most sensitivity analyses yielded findings that were consistent with those of the primary analysis, with the exception of two analyses that led to different point estimates. Specifically, using a less restrictive outcome definition consisting of a primary and secondary diagnosis of pneumonia led to a lower point estimate (HR 1.75, 95\% CI 1.42 to 2.16). In contrast, adjusting the model for high-dimensional propensity scores led to an increase in the HR (HR 2.33, 95\% CI 1.50 to 3.60$)$.

\section{DISCUSSION}

In this large population-based study, the use of ADT was associated with an $81 \%$ increased risk of hospitalisation for community-acquired pneumonia. An association was evident with $<6$ months of use, which remained with increasing durations of use. In contrast, past use of ADT was not associated with an increased risk of hospitalisation for community-acquired pneumonia. Overall, these findings remained consistent across several sensitivity analyses.
To our knowledge, two observational studies have investigated the association between the use of ADT and the risk of pneumonia. ${ }^{14} 15$ In one study, the use of GnRH agonists was associated with an increased risk of hospitalisation for pneumonia (HR $1.92,95 \%$ CI 1.10 to 3.36$).{ }^{14}$ In contrast, the second study observed less marked associations with community-acquired pneumonia (defined as a diagnosis from both inpatient and physician office visits) among those who used GnRH agonists for $>7$ months (7-11 months HR 1.09 , 95\% CI 1.04 to 1.15; $>11$ months HR 1.15 , 95\% CI 1.08 to 1.22 ). In contrast, the authors reported no associations with hospitalisations for pneumonia, determined from an inpatient diagnosis of pneumonia in the primary or secondary position. ${ }^{15}$ In addition, the authors reported associations with reductions in community-acquired pneumonia risk with orchiectomy (HR 0.80 , 95\% CI 0.72 to $0.90)$, but not for hospitalisation for community-acquired pneumonia. ${ }^{15}$ However, this study suffered from selection bias by the exclusion of those patients who underwent orchiectomy more than 6 months after diagnosis or received ADT more than 2 years after diagnosis. The authors also did not account for the time-varying nature of ADT, thus introducing immortal time bias. ${ }^{33}$ Specifically, this analysis misclassified unexposed persontime prior to ADT initiation during follow-up as exposed persontime. The discrepancies in results may also reflect various other methodological shortcomings including the potential 
Table 2 Crude and adjusted HRs for the association between the use of androgen deprivation therapy (ADT) and the risk of community-acquired pneumonia

\begin{tabular}{|c|c|c|c|c|c|}
\hline Exposure to ADT & Events & Person- years & Incidence rate* $(95 \% \mathrm{Cl})$ & Crude HR & Adjusted HR $(95 \% \mathrm{Cl}) \dagger$ \\
\hline Non-use & 147 & 38535 & $3.8(3.2-4.5)$ & 1.00 & 1.00 (reference) \\
\hline Current use & 364 & 30052 & $12.1(10.9-13.4)$ & 3.32 & $1.81(1.47-2.23)$ \\
\hline Past use & 110 & 17713 & $6.2(5.1-7.5)$ & 1.44 & $1.23(0.95-1.60)$ \\
\hline \multicolumn{6}{|l|}{ Duration of current use, months } \\
\hline$<6$ & 63 & 6506 & $9.7(7.4-12.4)$ & 2.62 & $1.73(1.23-2.42)$ \\
\hline $6-12$ & 45 & 5076 & $8.9(6.5-11.9)$ & 2.85 & $1.76(1.19-2.60)$ \\
\hline $13-18$ & 41 & 3610 & $11.4(8.2-15.4)$ & 4.29 & $2.45(1.64-3.67)$ \\
\hline $19-24$ & 30 & 2859 & $10.5(7.1-15.0)$ & 2.93 & $1.62(1.02-2.56)$ \\
\hline$\geq 25$ & 185 & 12001 & $15.4(13.3-17.8)$ & 3.69 & $1.79(1.39-2.30)$ \\
\hline \multicolumn{6}{|l|}{ ADT type } \\
\hline GnRH agonist alone & 248 & 21426 & $11.6(10.2-13.1)$ & 3.25 & $1.73(1.39-2.16)$ \\
\hline Oral antiandrogens alone & 43 & 3874 & $11.1(8.0-15.0)$ & 2.93 & $1.64(1.16-2.32)$ \\
\hline GnRH agonists and oral antiandrogens & 51 & 3575 & $14.3(10.6-18.8)$ & 3.66 & $2.18(1.56-3.03)$ \\
\hline Oestrogens alone & 5 & 130 & $38.5(12.5-89.8)$ & 8.83 & $4.50(1.82-11.17)$ \\
\hline Bilateral orchiectomy alone & 6 & 405 & $14.8(5.4-32.2)$ & 3.73 & $2.32(1.02-5.30)$ \\
\hline Other combinations & 11 & 643 & $17.1(8.5-30.6)$ & 4.08 & $2.47(1.33-4.61)$ \\
\hline
\end{tabular}

misclassification of hospital-acquired pneumonia as community-acquired, ${ }^{15} 16$ and the inclusion of patients with metastatic disease. ${ }^{14}$ In addition, both studies lacked information on potentially important confounders such as smoking status and influenza and pneumococcal vaccinations, and did not consider ADT pharmacotherapies other than GnRH agonists.

There are two possible biological mechanisms that may explain the observed association between the use of ADT and the risk of community-acquired pneumonia. First, androgen receptors are expressed on a number of immunological cells ${ }^{8}$ and are reported to stimulate the proliferation of granulocytic precursors $^{34}$ and neutrophil production. ${ }^{9-11}$ A recent study found that androgen receptor knockout mice had increased rates of neutropenia and were more susceptible to microbial infection. ${ }^{12}$ Moreover, exogenous androgens were reported to restore neutrophil counts in castrated mice. ${ }^{12}$ Additionally, ADT with antiandrogens could impair the adaptive immune response through interference with T-cell priming. ${ }^{13}$ Second, it has also been proposed that androgen deprivation may influence the risk of lower respiratory tract infections via morphological and biochemical changes to the cell membranes within the lung. ${ }^{6}{ }^{7}$ In addition, androgen deprivation has been shown to cause changes to the synthesis and secretion of phospholipids in pulmonary surfactant. ${ }^{6} 7$
Figure 2 Restricted cubic spline of duration of androgen deprivation therapy (ADT) use. Smoothed restricted spline curve of the adjusted HR of hospitalisation of community-acquired pneumonia (HCAP) (solid line) and $95 \%$ confidence limits (dashed lines) as a function of cumulative duration of ADT use.

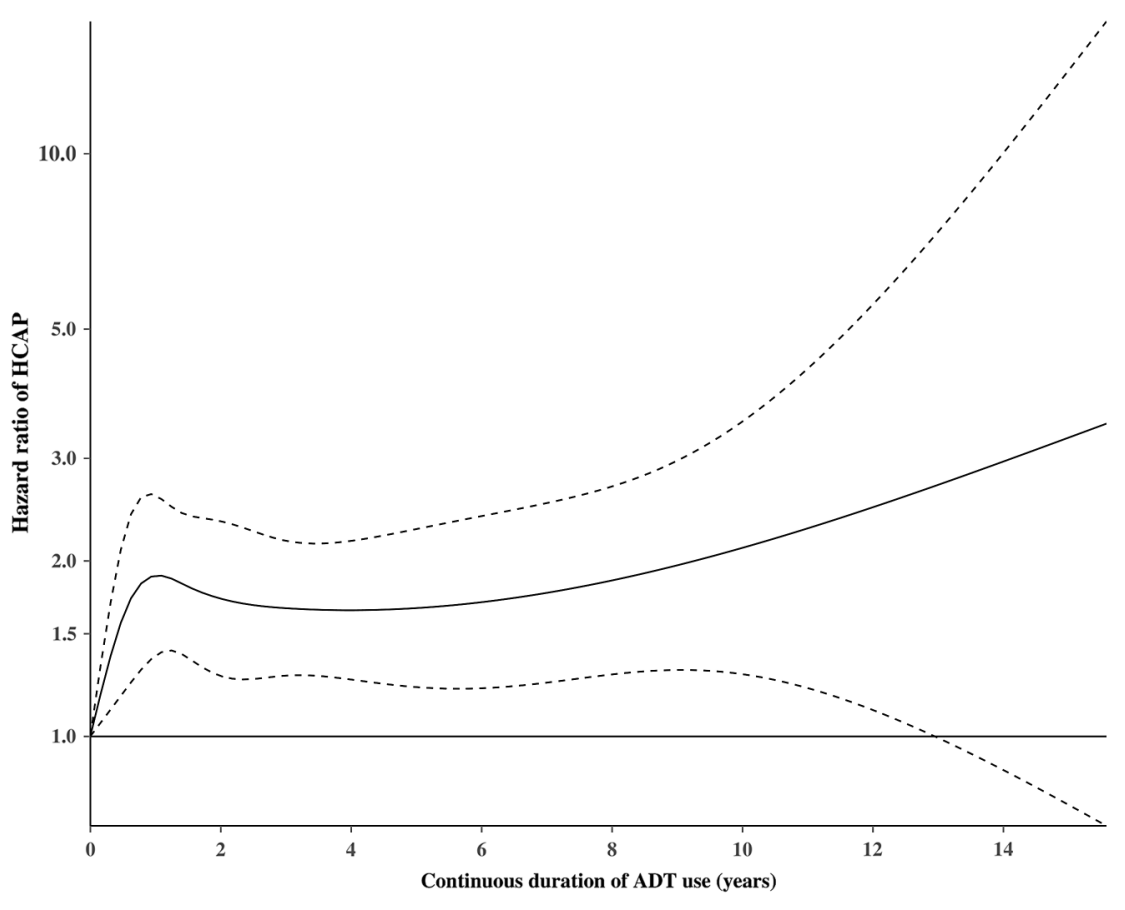

Hicks BM, et al. Thorax 2017;72:628-634. doi:10.1136/thoraxjnl-2016-209512 
Figure 3 Sensitivity analyses for the association between current use of androgen deprivation therapy and the risk of hospitalisation for community-acquired pneumonia in patients with prostate cancer. ${ }^{*}$ Hospitalisation for community-acquired pneumonia (HCAP) included those with a diagnosis for pneumonia in the primary or secondary position within tHospital-acquired pneumonia included pneumonia diagnoses $>2$ days after hospital admission and up to 14 days after discharge. the first two days of admission.

Analyses

HR $(95 \% \mathrm{Cl})$

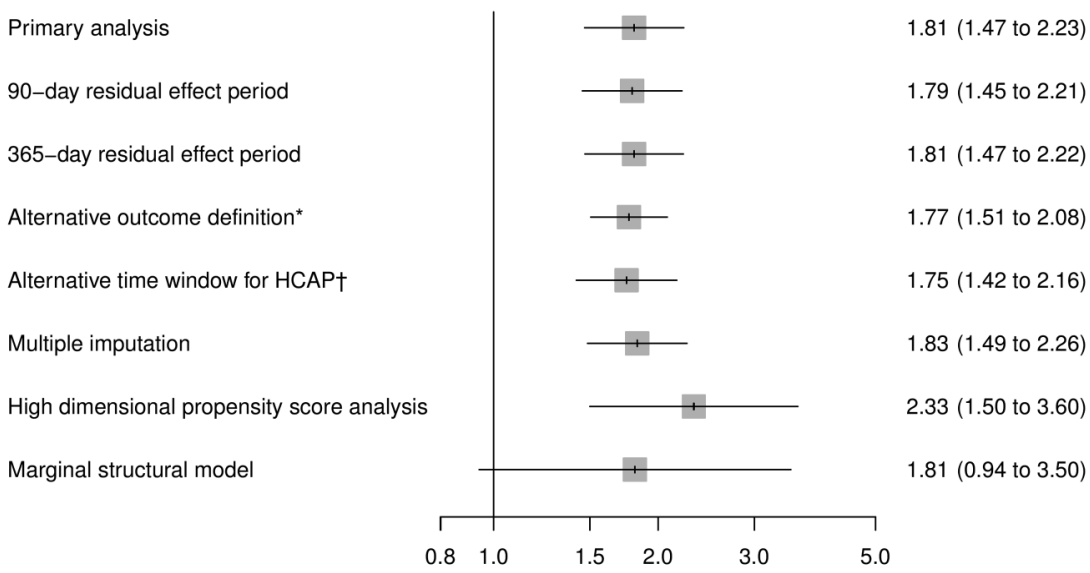

\section{Strengths and limitations}

This study had a number of strengths. This was a large population-based cohort study of 20310 patients with prostate cancer, followed for up to 18 years. This contrasts to previous studies that had a maximum follow-up of 2 years. ${ }^{14} 15$ Additionally, the use of ADT was modelled as a time-dependent variable (thereby eliminating immortal time bias ${ }^{33}$ ), and the use of the CPRD allowed us to adjust for a number of potentially important confounders such as BMI, smoking status and comorbidities.

This study does have some limitations. First, prescriptions recorded in the CPRD represent those written by general practitioners and not those issued by specialists. While the decision to initiate ADT is usually made by specialists, general practitioners typically follow through with the suggested treatment regimen within the UK healthcare system. Indeed, this is reflected by the high prevalence of ADT use in our cohort (58.7\%), which is similar to ADT utilisation rates reported in the USA. ${ }^{35}$ However, it is possible that the initial ADT prescriptions issued by the specialists were not captured in the CPRD, leading to a possible underestimation of the observed association. Second, while defining the outcome as a hospitalisation for communityacquired pneumonia likely maximised the specificity of the event definition, it did not consider pneumonias not requiring inpatient care, thus limiting the generalisability of our findings to severe community-acquired pneumonia. Third, given the observational nature of the study, residual confounding from unmeasured or unknown variables is possible. In particular, it was not possible to adjust the models for tumour stage and grade as this information is not recorded in the CPRD. To mitigate this issue, we conducted a number of sensitivity analyses that led to consistent findings. These included the use of highdimensional propensity scores and marginal structural models. The high-dimensional propensity score allowed for the inclusion of a large number of covariates at cohort entry, which comprised 20 predefined covariates plus an additional 500 empirically defined covariates ascertained from patients' data. This may reduce residual confounding as these variables may be proxies for unmeasured covariates, such as those related to disease severity. In addition, the marginal structural model adjusted for important time-dependent variables known to be correlated with disease progression that were measured in our data set during the follow-up period. These included radiation therapy, radical prostatectomy, incident metastases, receipt of chemotherapy, use of oral bisphosphonates, as well as other time- dependent confounders such as seasonality and receipt of influenza and pneumococcal vaccinations.

\section{CONCLUSION}

The results of this study indicate that the use of ADT is associated with an increased risk of hospitalisation for communityacquired pneumonia. These results are of importance as community-acquired pneumonia is of particular concern among patients with cancer for whom severe pneumonia can result in high mortality rates. Given these findings, physicians should exercise caution when considering ADT in low-risk patients for whom the benefits remain uncertain. the Fonds de Recherche du Québec-Santé.

Contributors All authors conceived and designed the study, analysed and interpreted the data, and critically revised the manuscript for important intellectual content. LA acquired the data. BMH, HY, FB, PE and LA did the analyses. BMH wrote the manuscript and all authors participated in the interpretation of the results and critical revision of the manuscript. LA is the guarantor.

Funding This study was funded by a Foundation Grant from the Canadian Institutes of Health Research.

Competing interests None declared.

Ethics approval The study protocol was approved by the Independent Scientific Advisory Committee of the Clinical Practice Research Datalink (protocol number 16_144) and by the Research Ethics Board of Jewish General Hospital, Montreal, Quebec, Canada.

Provenance and peer review Not commissioned; externally peer reviewed.

\section{REFERENCES}

1 Gilbert SM, Kuo YF, Shahinian VB. Prevalent and incident use of androgen deprivation therapy among men with prostate cancer in the United States. Urol Oncol 2011;29:647-53.

2 National Institute for Health and Clinical Excellence. Prostate cancer: diagnosis and management. 2014. https://www.nice.org.uk/guidance/cg175/chapter/ 1-Recommendations\#metastatic-prostate-cancer (accessed 10 Apr 2016).

3 Reichert ZR, Hussain M. Androgen receptor and beyond, targeting androgen signaling in castration-resistant prostate cancer. Cancer J 2016;22:326-9.

4 Nguyen PL, Alibhai SMH, Basaria $\mathrm{S}$, et al. Adverse effects of androgen deprivation therapy and strategies to mitigate them. Eur Urol 2015;67:825-36.

5 Crawford ED, Moul JW. ADT risks and side effects in advanced prostate cancer. Oncology (Williston Park) 2015;29:55-8, 65-6.

6 Ojeda MS, Gomez N, Giménez MS. Androgen regulation of lung lipids in the Male rat. Lipids 1997;32:57-62.

7 Ojeda MS, Gómez NN, Gil E, et al. Morphologic and biochemical changes in Male rat lung after surgical and pharmacological castration. Braz J Med Biol Res 2000;33:279-85.

8 Mantalaris A, Panoskaltsis N, Sakai Y, et al. Localization of androgen receptor expression in human bone marrow. J Pathol 2001;193:361-6.
Acknowledgements LA is the recipient of a Chercheur-Boursier career award from 
9 Alter BP. Fanconi's anemia. Current concepts. Am J Pediatr Hematol Oncol 1992;14:170-6.

10 Ibáñez L, Jaramillo AM, Ferrer A, et al. High neutrophil count in girls and women with hyperinsulinaemic hyperandrogenism: normalization with metformin and flutamide overcomes the aggravation by oral contraception. Hum Reprod 2005:20:2457-62.

11 Rawbone RG, Bagshawe KD. Anabolic steroids and bone marrow toxicity during therapy with methotrexate. Br J Cancer 1972:26:395-401.

12 Chuang K-H, Altuwaijri S, Li G, et al. Neutropenia with impaired host defense against microbial infection in mice lacking androgen receptor. J Exp Med 2009:206:1181-99.

13 Pu Y, Xu M, Liang Y, et al. Androgen receptor antagonists compromise T cell response against prostate cancer leading to early tumor relapse. Sci Trans/ Med 2016;8:333ra47.

14 Chung S-D, Liu S-P, Lin H-C, et al. Increased risk of pneumonia in patients receiving gonadotropin-releasing hormone agonists for prostate cancer. PLOS ONE 2014;9: e101254.

15 Schmid M, Hanske J, Ravi P, et al. Relationship between androgen deprivation therapy and community-acquired respiratory infections in patients with prostate cancer. Int J Urol 2016;23:305-11.

16 Kaplan V, Clermont G, Griffin MF, et al. Pneumonia. Still the Old Man's Friend? Arch Intern Med 2003;163:317.

17 Rabello LSCF, Silva JRL, Azevedo LCP, et al. Clinical outcomes and microbiological characteristics of severe pneumonia in cancer patients: a prospective cohort study. PLOS ONE 2015;10:e0120544.

18 National Health Service. Read Codes. http://systems.digital.nhs.uk/data/uktc/readcodes

19 Authority NHS Buisness Service Authority. Dictionary of Medicines and Devices. http://www.nhsbsa.nhs.uk/PrescriptionServices/1121.aspx (accessed 10 Apr 2016).

20 Jick H, Jick SS, Derby LE. Validation of information recorded on general practitioner based computerised data resource in the United Kingdom. BMJ 1991;302:766-8.

21 Herrett E, Thomas SL, Schoonen WM, et al. Validation and validity of diagnoses in the General Practice Research Database: a systematic review. Br J Clin Pharmacol 2010:69:4-14.
22 Herrett E, Gallagher AM, Bhaskaran K, et al. Data resource profile: Clinical Practice Research Datalink (CPRD). Int J Epidemiol 2015:44:827-36.

23 Bong GW, Clarke HS, Hancock WC, et al. Serum testosterone recovery after cessation of long-term luteinizing hormone-releasing hormone agonist in patients with prostate cancer. Urology 2008;71:1177-80.

24 Kaku H, Saika T, Tsushima T, et al. Time course of serum testosterone and luteinizing hormone levels after cessation of long-term luteinizing hormone-releasing hormone agonist treatment in patients with prostate cancer. Prostate 2006;66:439-44.

25 Mann AG, Mangtani P, Russell CA, et al. The impact of targeting all elderly persons in England and Wales for yearly influenza vaccination: excess mortality due to pneumonia or influenza and time trend study. BMJ Open 2013;3:e002743.

26 World Health Organisation. Review of the 2014-2015 influenza season in the northern hemisphere. Wkly Epidemiol Rec 2015;90:281-96.

27 Greenland S. Dose-response and trend analysis in epidemiology: alternatives to categorical analysis. Epidemiology 1995;6:356-65.

28 Heinzl H, Kaider A, Zlabinger G. Assessing interactions of binary time-dependent covariates with time in cox proportional hazards regression models using cubic spline functions. Stat Med 1996;15:2589-601.

29 Schafer JL. Analysis of Incomplete Multivariate Data. Chapman and Hall, 1997.

30 Rubin DB, ed. Multiple Imputation for Nonresponse in Surveys. Hoboken, NJ, USA: John Wiley \& Sons, Inc., 1987.

31 Robins JM, Hernán MA, Brumback B. Marginal structural models and causal inference in epidemiology. Epidemiology 2000;11:550-60.

32 Hernán MA, Brumback B, Robins JM. Marginal structural models to estimate the causal effect of zidovudine on the survival of HIV-positive men. Epidemiology 2000:11:561-70

33 Suissa S. Immortal time bias in pharmaco-epidemiology. Am J Epidemiol 2008;167:492-9.

34 Udupa KB, Reissmann KR. Acceleration of granulopoietic recovery by androgenic steroids in mice made neutropenic by cytotoxic drugs. Cancer Res 1974:34:2517-20.

35 Keating NL, O'Malley AJ, Smith MR. Diabetes and cardiovascular disease during androgen deprivation therapy for prostate cancer. J Clin Oncol 2006;24:4448-56. 